\title{
Antivitamin K Drugs in Stroke Prevention
}

\author{
Domenico Di Raimondo*, Antonio Tuttolomondo, Giuseppe Licata ${ }^{\circ}$ and Antonio Pinto
}

\author{
U.O.C. di Medicina Vascolare - ${ }^{\circ}$ U.O.C. di Medicina Interna e Cardioangiologia, Dipartimento Biomedico di Medicina \\ Interna e Specialistica, Università degli Studi di Palermo, Palermo, Italy
}

\begin{abstract}
Among the different subtypes of ischaemic strokes, almost $20 \%$ are of cardiac origin. Different are the causes of cardioembolic stroke, but the most common is the atrial fibrillation, a supraventricular arrhythmia.

Appropriate use of antiplatelet drugs and anticoagulants after transient ischaemic attack (TIA) or ischaemic stroke depends on whether the underlying cause is cardioembolic or of presumed arterial origin.

Adequate antiplatelet therapy is recommended for secondary prevention after cerebral ischaemia of presumed arterial origin, whether for patients with TIA and ischaemic stroke of cardiac origin, mainly due to atrial fibrillation. Vitamin K antagonists (VKAs) are highly effective in preventing recurrent ischaemic stroke but have important limitations and are thus underused.

Current guidelines still regard Vitamin K Antagonists at INR 2.0-3.0 to be the standard treatment after cerebral ischaemia of cardiac origin for patients who can tolerate them. In this setting antiplatelet therapy provides an alternative when oral anticoagulation is contraindicated or when patient choice or compliance limits choice of therapy, but is much less effective than VKAs. Recent trial data performed with new anticogulants such as the factor Xa and thrombin inhibitors will need to be taken into account, in order to prevent several of the clinical problems actually related to VKAs use.
\end{abstract}

Keywords: ??????????????????????

\section{INTRODUCTION}

Among the different subtypes of ischaemic strokes, almost $20 \%$ are of cardiac origin. Different are the causes of cardioembolic stroke, but the most common is the atrial fibrillation (AF), a supraventricular arrhythmia. AF is associated with a four- to fivefold increase in the risk of ischemic stroke, and $15 \%$ of all ischemic strokes are caused by AF. Importantly, this proportion increases substantially with age [1].

Strategies to reduce risk of recurrence of the cerebral ischemia after a transient ischaemic attack (TIA) or ischaemic stroke depend, among other interventions, on appropriate use of antiplatelet drugs and anticoagulants after the identification of the underlying cause of the first event: that is, in the greatest part of ischemic strokes, cardioembolic or due to atherosclerotis of the arteries [2].

The standard therapy to prevent stroke and systemic thromboembolism associated with cardiac origin has been anticoagulation with a coumarin derivative (or vitamin $\mathrm{K}$ antagonist [VKA]). However, coumarins have a narrow therapeutic index and require ongoing frequent monitoring. Newer antithrombotic agents are destined to provide stroke prevention therapy without the problems inherent in frequent monitoring of the International Normalized Ratio (INR).

*Address correspondence to this author at the Dipartimento Biomedico di Medicina Interna e Specialistica, Università degli Studi di Palermo, Palermo, Italy; Tel: ++39-091/6552180; Fax: +39-091/6552285;

E-mail: diraimondo@unipa.it

$1570-1611 / 13 \$ 58.00+.00$
Aim of this review is to provide a brief evaluation, in the age of new oral anticoagulants, of available data about effectiveness of vitamin $\mathrm{K}$ antagonists therapy for the prevention of stroke and to discuss the expected benefit of use of antiplatelet drugs in these patients when VKAs are contraindicated or the compliance of the patients does not allow the use of these compounds.

\section{MAIN CHARACTERISTICS OF VITAMIN K AN- TAGONISTS (VKAS)}

The available anticoagulant drugs inhibit various components of the clotting cascade. Coumarins cause $\gamma$ carboxylation and inhibition of vitamin $\mathrm{K}$ - dependent clotting factors (factors II, VII, IX, and X) and vitamin K-dependent anticoagulant factors (protein $\mathrm{C}$ and $\mathrm{S}$ ) [3]. Thus, the biological effect of coumarins is directly related to the normal half-life of these factors and, generally, the full anticoagulant effect is not seen for 4 to 7 days after commencing daily therapy [4]. As a consequence of this gap, usually bridging therapy with another anticoagulant drug, usually low molecular weight heparins (LMWHs), is associated to guarantee a potential anticoagulant effect until therapeutic INR target is reached and the level of VKAs activity becomes therapeutic.

Nowadays, VKAs are the standard-of-care oral anticoagulants for long-term stroke prevention in established cardioembolic stroke.

The risk of major bleeding is significantly higher with coumarins compared with aspirin (2.2 vs 1.3 events per 100 patient-years) and risk increases with the INR value [5]. An- 
tiplatelet use, advanced age, supratherapeutic INR, and prior stroke increase the risk of intracranial hemorrhage (ICH) [6]. Advancing age has been presumed to be a risk factor for bleeding during anticoagulation perhaps because of the accumulation of other risk factors such as stroke and more widespread use of antiplatelet agents.

When needed, rapid reversal of INR might be obtained through use of vitamin $\mathrm{K}$ infusion, fresh frozen plasma (FFP), and/or Factor VIIa. These interventions rapidly reverse INR but they have not yet been shown to completely reverse all aspects of VKA-associated hemorrhage [7].

A usual clinical problem observed by clinicians who currently use VKAs is the interactions of these compounds with several other drugs [8] and the different therapeutic effect observed according to various degree of diet-derived vitamin $\mathrm{K}$.

Another common clinical problem concerns the optimal behavior for patients who are chronically anticoagulated for $\mathrm{AF}$, but require a minor or major surgical procedure. Practice is highly variable in this circumstance. Some physicians will bridge patients with LMWH until the day of the procedure, and restart thereafter. Others will simply stop VKAs a week before and restart thereafter. A result of imperfect VKAs stopping and restarting is the rather common story of presentation for cardioembolic stroke of a patient who stopped their VKA and had a colonoscopy yesterday or is due for a small biopsy but never gets there because they are admitted with a stroke.

There are reasonable data to suggest that minor procedures such as tooth extractions, skin biopsies, pacemaker placement, and cataract removal do not require cessation of VKAs [8]. This information is not widely known and many surgeons refuse to perform such procedures while patients are on VKAs. Education is required. Practically, invasive procedures will require the cessation of VKAs but it remains unknown if bridging with LMWH is appropriate. It seems likely that a risk stratification process could be developed for this situation. Further data are needed to better standardize behavior of clinicians in these situations to provide maximum protection to anticoagulated patients.

\section{ANTIPLATELET VS ANTICOAGULANTS IN SEC- ONDARY PREVENTION AFTER CEREBRAL ISCHAEMIA OF ARTERIAL ORIGIN}

As widely discussed in another review in this journal, adequate antiplatelet therapy is recommended for secondary prevention after cerebral ischaemia of presumed arterial origin. This evidence derived mainly from the results of three large trials [9-11], that investigated whether vitamin $\mathrm{K}$ antagonists (VKAs) would be more effective than aspirin for this indication.

The SPIRIT trial [9] compared $30 \mathrm{mg}$ daily aspirin against warfarin (INR target 3.0-4.5) in an open randomized trial of over a thousand stroke patients on sinus rhythm. Due to a high rate of hemorrhagic complications in the anticoagulation group the study was terminated prematurely. Major systemic and intracranial hemorrhage rates were $8 \%$ in the warfarin group versus $1 \%$ in the aspirin group (hazard ratio [HR], 9.3; 95\% CI, 4.0-22). The occurrence of ischemic events was similar in both groups $(4.1 \%$ in both groups; HR, 1.03 ; 95\% CI, 0.6-1.75) including vascular death, nonfatal ischemic stroke, and nonfatal MI.

More conservative INR targets have also failed to establish an advantage of warfarin over aspirin as demonstrated by the WARSS trial [10], which tested INRs between 1.4 and 2.8. 2.206 Patients with noncardioembolic stroke were included in this trial. The results show similarity in stroke recurrence and death in both groups, with an excess of systemic bleeds in those allocated to warfarin.

The ESPRIT trial [11] randomized 1,068 patients with prior stroke or TIA to warfarin (INR target 2.0-3.0) or aspirin, 30-325 mg, per day. Treatment was randomized but open; outcome event assessment was blinded. Event this study was prematurely terminated due to the fact that randomization to the aspirin or aspirin plus dipyridamole intervention was determined to be superior. There were no significant differences in terms of primary outcome (stroke, MI, vascular death, or major bleeds) in the aspirin group compared with warfarin, with an $18 \%$ event rate in the aspirin and $19 \%$ in the warfarin group (HR, 1.02; 95\% CI, 0.771.35). There was a nonsignificant trend toward a lower rate of ischemic stroke in the warfarin group (7.6\% vs. $10.0 \%$; HR, 0.76; 95\% CI, 0.51-1.15), which was offset by a significantly higher rate of major bleeding in this group $(8.4 \%$ vs. $3.4 \%$; HR, 2.56; 95\% CI, 1.48-4.43)

The absence of benefit, irrespective of the intensity of anticoagulation used, with a significant increase in risk of intracerebral haemorrhage on high-intensity anticoagulation (international normalised ratio $[\mathrm{INR}] \geq 3$ ) conditioned that the use of VKAs for this indication is not advised $[12,13]$.

A rather different setting is the one of the WASID trial [14], in which high aspirin dose (1,3 g/day) was compared to dose-adjusted warfarin with an INR target between 2.0 and 3.0 even in secondary prevention after a prior stroke in subjects with atherosclerotic stenosis.

Five hundred sixty-nine patients with $\geq 50 \%$ stenosis were enrolled. There was no significant difference in recurrent stroke rate, with $22 \%$ recurrence over 1.8 years of follow-up in the aspirin group and $22 \%$ in the warfarin group (HR, 1.04; 95\% CI, 0.73-1.48). Enrollment was stopped early due to high rates of adverse events among the patients receiving warfarin, including death $(4.3 \%$ in the aspirin group vs. $10 \%$ in the warfarin group; HR, 0.46; 95\%CI, 0.23-0.90; $\mathrm{P}=0.02$ ), major hemorrhage (3\% vs. 8\%; HR, $0.39 ; 95 \% \mathrm{CI}, 0.18-0.84 ; \mathrm{P}=0.01)$, and $\mathrm{MI}$ and sudden death (3\% vs. $7 \%$; HR, 0.40; 95\% CI, 0.18-0.91; P=0.02). So, despite the fact that new anticoagulants actually under development might provide greater benefits also for this indication, actually VKAs, also with more conservative INR targets, are not indicated in the secondary prevention of stroke with ascertained atherosclerotic origin.

\section{ANTICOAGULANTS IN PRIMARY AND SECON- DARY PREVENTION OF CEREBROVASCULAR EVENTS IN PATIENTS WITH ATRIAL FIBRILLA- TION}

Randomized clinical trials have firmly established the value of antithrombotic therapies for reducing the risk of 
stroke in patients with previous cardioembolic stroke. Among the various cardiomyopathies, Atrial fibrillation (AF) is the most common source of cardiogenic brain embolism [15].

The prevalence of AF increases sharply with age: six percent of the population > 65 years of age has AF [16], and with increasing life expectancy, cardioembolic events are likely to become even more important.

Approximately $70 \%$ of patients with AF have nonvalvular atrial fibrillation (NVAF) associated with underlying non-rheumatic heart disease, $20 \%$ have underlying rheumatic heart disease and $10 \%$ have lone atrial fibrillation without obvious structural heart disease [17].

As reported in the introduction, about $20 \%$ of all TIAs and ischaemic strokes have a cardiac origin, most commonly atrial fibrillation. There is much variation, however, in the risk of stroke in patients with AF, which can be assessed with risk scoring systems such as the CHADS2-score [18]. However, a general assessment of risk estimates that patients with NVAF who present with TIA or stroke have a n overall risk of recurrent stroke of $12 \%$ in the first year and $5 \%$ per annum thereafter [19].

Efficacy of VKAs in prevention of embolism in subjects with nonvalvular AF is well established from many trials (AFASAK, BAATAF, SPINAF, CAFA) mainly comparing warfarin vs. placebo [20-23]. These trial found a relative risk reduction of $2.5 \%$ to $4.7 \%$ per year for ischemic stroke and absolute stroke rate reduction of $33 \%$ to $86 \%$.

The AFASAK trial, tested the hypothesis of effectiveness of anticoagulation therapy with warfarin in prevention of thromboembolic complications in patients with chronic nonrheumatic AF in comparison with placebo and aspirin 75 $\mathrm{mg}$ /day [20]. This trial enrolled 1007 outpatients with chronic non-rheumatic AF: 335 received anticoagulation with warfarin openly, and in a double-blind study 336 received aspirin $75 \mathrm{mg}$ once daily and 336 placebo. Each patient was followed up for 2 years or until termination of the trial. The primary endpoint was a thromboembolic complication (stroke, transient cerebral ischaemic attack, or embolic complications to the viscera and extremities). The secondary endpoint was death. The incidence of thromboembolic complications and vascular mortality were significantly lower in the warfarin group than in the aspirin and placebo groups, which did not differ significantly. 5 patients on warfarin had thromboembolic complications compared with 20 patients on aspirin and 21 on placebo. 21 patients on warfarin were withdrawn because of non-fatal bleeding complications compared with 2 on aspirin and none on placebo.

The BAATAF Trial [21], was an unblinded, randomized, controlled trial of long-term, low-dose warfarin therapy (target prothrombin-time ratio, 1.2 to 1.5 ) in patients with nonrheumatic AF, controlled with placebo or aspirin. A total of 420 patients entered the trial (212 in the warfarin group and 208 in the control group) and were followed for an average of 2.2 years. Prothrombin times in the warfarin group were in the target range 83 percent of the time. Only 10 percent of the patients assigned to receive warfarin discontinued the drug permanently. There were 2 strokes in the warfarin group (incidence, 0.41 percent per year) as compared with 13 strokes in the control group (incidence, 2.98 percent per year), for a reduction of 86 percent in the risk of stroke (warfarin:control incidence ratio $=0.14 ; 95$ percent confidence interval, 0.04 to $0.49 ; \mathrm{P}=0.0022$ ). Long-term VKA use was effective in preventing stroke in patients with non-rheumatic AF.

The SPINAF trial [22], was a randomized, double-blind, placebo-controlled study to evaluate low-intensity anticoagulation with warfarin (prothrombin-time ratio, 1.2 to 1.5 ) in 571 men with chronic nonrheumatic AF (525 patients had not previously had a cerebral infarction, whereas 46 patients had previously had such an event)., controlled vs placebo. The primary end point was cerebral infarction; secondary end points were cerebral hemorrhage and death. Among the patients with no history of stroke, cerebral infarction occurred in 19 of the 265 patients in the placebo group during an average follow-up of 1.7 years (4.3 percent per year) and in 4 of the 260 patients in the warfarin group during an average follow-up of 1.8 years ( 0.9 percent per year). The reduction in risk with warfarin therapy was 0.79 (95 percent confidence interval, 0.52 to $0.90 ; \mathrm{P}=0.001)$. The annual event rate among the 228 patients over 70 years of age was 4.8 percent in the placebo group and 0.9 percent in the warfarin group (risk reduction, $0.79 ; \mathrm{P}=0.02$ ). The only cerebral hemorrhage occurred in a 73-year-old patient in the warfarin group. Other major hemorrhages, all gastrointestinal, occurred in 10 patients: 4 in the placebo group, for a rate of 0.9 percent per year, and 6 in the warfarin group, for a rate of 1.3 percent per year. There were 37 deaths that were not preceded by a cerebral end point-- 22 in the placebo group and 15 in the warfarin group (risk reduction, 0.31; $\mathrm{P}=0.19$ ). Cerebral infarction was more common among patients with a history of cerebral infarction (9.3 percent per year in the placebo group and 6.1 percent per year in the warfarin group) than among those without such a history. In this study also a lower INR target seems to guarantee protection against thromboembolic events in patients with AF.

The Canadian Atrial Fibrillation Anticoagulation Study [23] was a randomized double-blind placebo-controlled trial to assess the potential of warfarin to reduce systemic thromboembolism and its inherent risk of hemorrhage. As a result of the publication of two other "positive" studies of similar design and objective, this study was stopped early before completion of its planned recruitment of 630 patients. There were 187 patients randomized to warfarin and 191 to placebo. Permanent discontinuation of study medication occurred in $26 \%$ of warfarin-treated and $23 \%$ of placebotreated patients. The target range of the international normalized ratio was 2 to 3 . The annual rates of the primary outcome event cluster were $3.5 \%$ in warfarin-treated and $5.2 \%$ in placebo-treated patients, with a relative risk reduction of $37 \%$ (95\% confidence limits, $-63.5 \%, 75.5 \%, p=0.17$ ), confirming effectiveness of VKA use vs placebo in this setting.

Current guidelines still regard VKAs at INR 2.0-3.0 to be the standard treatment after cerebral ischaemia of cardiac origin for patients who can tolerate them [24,25].

Warfarin reduces the risk of recurrent stroke or systemic embolism by about $61 \%$ (95\% CI 37-75\%) compared with control in AF patients with recent TIA or ischaemic stroke. This proportional risk reduction is consistent with that ob- 
served for the prevention of first-ever stroke among individuals with AF, including the elderly [26,27].

Warfarin also increases the odds of major extracranial haemorrhage (OR 4.3 [95\% CI 1.5-12.1]) [27].

The decision to prescribe warfarin, and the net clinical benefit of warfarin, is based on an accurate assessment of the likely absolute annual risk of stroke without warfarin, and whether the likely benefits of warfarin (a two-thirds reduction in absolute stroke risk) are likely to outweigh the risks of bleeding associated with warfarin use [28].

More recently, several randomized trials have also been conducted comparing new anticoagulants compounds such as direct Factor Xa inhibitors (Apixaban, Rivaroxaban, Edoxaban, Betrixaban) [29] or direct Thrombin (F II) Inhibitors (Ximelagatran, Dabigatran) [30-33] to high quality adjusted-dose warfarin in persons with atrial fibrillation. The relationship between new and old anticoagulant, the advantages of oral administration, the better pharmacokinetic profile, the rapid onset of the pharmacologic action, the lower interactions with food and drugs, and the predictivity of anticoagulant response without need of coagulation monitoring are matters of absolute interest and strict actuality, but these arguments exceed the aim of the present review.

\section{ANTIPLATELET VS ANTICOAGULANTS IN PRI- MARY PREVENTION IN PATIENTS WITH ATRIAL FIBRILLATION AND IN SECONDARY PREVEN- TION AFTER A CARDIOEMBOLIC STROKE}

As showed before oral anticoagulants remain the treatment of choice for secondary prevention of cardioembolic stroke. However, antiplatelet therapy provides an alternative when oral anticoagulation is contraindicated or when patient choice or compliance limits choice of therapy. Infact, despite the efficacy and affordability of warfarin, many patients with cardioembolic ischaemic stroke or TIA are not treated with warfarin because it is perceived to be inconvenient or hazardous. For those who are treated, the long-term efficacy and safety of warfarin depends on maintaining a narrow range of anticoagulation intensity (INR 2.0-3.0). However, as previously underlined, this measure is often compromised by the patient's dietary intake, exposure to other drugs, and coexisting illnesses. Consequently, many drug-compliant patients are not well controlled. In recent, carefully monitored trials, patients treated with warfarin spent only $63-68 \%$ of the time in the therapeutic INR range [34-36]. However, in many clinical settings, time within this INR range is much lower [37], and many patients discontinue treatment [38]. Consequently, alternative antithrombotic regimens of similar efficacy to warfarin, but without the limitations, are needed, and antiplatelet drugs are perceived as an effective alternative.

Nevertheless of this, the superiority of warfarin over combination antiplatelet therapy for stroke prevention in nonvalvular atrial fibrillation has been shown in several trials. A meta-analysis of 29 trials with 28,044 patients showed a $64 \%$ reduction in stroke in the warfarin group compared with $22 \%(95 \% \mathrm{CI}, 0.49-0.74)$ in the antiplatelet groups (95\% CI, 0.06-0.35) for a RR reduction of 39\% (95\% CI, $0.22-0.52)[26,39]$.
Superiority of VKAs was demonstrated in comparison to different antiplatelet compounds either administrated in single or in combination therapy.

Adjusted-dose warfarin reduces stroke by $\sim 45 \%$ as compared with aspirin [5].

The comparison between VKAs and aspirin in secondary prevention of stroke in patients with AF showed that adjusted-dose warfarin is significantly more effective than antiplatelet therapy for preventing recurrent stroke (OR 0.49 [95\% CI 0.33-0.72]) [26].

Although major extracranial bleeding complications occurred more often in patients on anticoagulants (OR 5.2 [2·1-12.8]), the absolute difference was small (1.9\%) [39].

The absolute benefit of oral anticoagulation (versus antiplatelet therapy) increases as patients with AF get older because stroke risk increases with age and the relative efficacy of oral anticoagulation therapy to prevent ischaemic stroke does not change. However, the relative efficacy of antiplatelet therapy to prevent ischaemic stroke seems to decrease as patients with AF get older.

The SPAF I trial [40], was a multicenter, randomized trial, compared $325 \mathrm{mg} /$ day aspirin (double-blind) or warfarin with placebo for prevention of ischemic stroke and systemic embolism (primary events), and included 1,330 inpatients and outpatients with constant or intermittent AF. During a mean follow-up of 1.3 years, the rate of primary events in patients assigned to placebo was $6.3 \%$ per year and was reduced by $42 \%$ in those assigned to aspirin (3.6\% per year; $\mathrm{p}=0.02 ; 95 \%$ confidence interval, $9-63 \%)$. In the subgroup of warfarin-eligible patients (most less than 76 years old), warfarin dose-adjusted to prolong prothrombin time to 1.3fold to 1.8 -fold that of control reduced the risk of primary events by $67 \%$ (warfarin versus placebo, $2.3 \%$ versus $7.4 \%$ per year; $\mathrm{p}=0.01 ; 95 \%$ confidence interval, 27-85\%). Primary events or death were reduced $58 \%(\mathrm{p}=0.01)$ by warfarin and $32 \%(\mathrm{p}=0.02)$ by aspirin. The risk of significant bleeding was $1.5 \%, 1.4 \%$, and $1.6 \%$ per year in patients assigned to warfarin, aspirin, and placebo, respectively.

Aspirin and warfarin are both effective in reducing ischemic stroke and systemic embolism in patients with atrial fibrillation. Because warfarin-eligible patients composed a subset of all aspirin-eligible patients, the magnitude of reduction in events by warfarin versus aspirin cannot be compared. Because of too few events occurred in warfarineligible patients to directly assess the relative benefit of aspirin compared with warfarin, the trial was continued to address this issue.

Due to the lack of direct comparison of warfarin with aspirin In the first SPAF-I study, caused by the small number of thromboembolic events in the follow-up, SPAF-II [41] aimed to address this issue and also to assess the differential effects of the two treatments according to age. We compared warfarin (prothrombin time ratio 1.3-1.8, international normalised ratio 2.0-4.5) with aspirin $325 \mathrm{mg}$ daily for prevention of ischaemic stroke and systemic embolism (primary events) in two parallel randomised trials involving 715 patients aged 75 years or less and 385 patients older than 75 ; we sought reductions in the absolute rate of primary events 
by warfarin compared with aspirin of $2 \%$ per year and $4 \%$ per year, respectively. In the younger patients, warfarin decreased the absolute rate of primary events by $0.7 \%$ per year (95\% CI-0.4 to 1.7). The primary event rate per year was $1.3 \%$ with warfarin and $1.9 \%$ with aspirin (relative risk [RR] $0.67, \mathrm{p}=0.24$ ). The absolute rate of primary events in lowrisk younger patients (without hypertension, recent heart failure, or previous thromboembolism) on aspirin was $0.5 \%$ per year (95\% CI 0.1 to 1.9 ). Among older patients, warfarin decreased the absolute rate of primary events by $1.2 \%$ per year $(95 \%$ CI-1.7 to 4.1$)$. The primary event rate per year was $3.6 \%$ with warfarin and $4.8 \%$ with aspirin (RR $0.73, \mathrm{p}=$ $0.39)$. In this older group, the rate of all stroke with residual deficit (ischaemic or haemorrhagic) was $4.3 \%$ per year with aspirin and $4.6 \%$ per year with warfarin (RR 1.1). Warfarin may be more effective than aspirin for prevention of ischaemic stroke in patients with atrial fibrillation, but the absolute reduction in stroke rate by warfarin is small. Younger patients without risk factors had a low rate of stroke when treated with aspirin. In older patients the rate of stroke (ischaemic and haemorrhagic) was substantial, irrespective of which agent was given. Patient age and the inherent risk of thromboembolism should be considered in the choice of antithrombotic prophylaxis for patients with atrial fibrillation.

The European Atrial Fibrillation Trial (EAFT) [19] compared the outcome of treatment with oral anticoagulants, aspirin or placebo in patients with persistent or paroxysmal NVAF who had experienced a cardioembolic TIA or minor ischaemic stroke in the preceding 3 months. Patients with no contraindications to VKAs were randomly allocated to VKA (INR 2.5-4.0), aspirin (300 mg daily), or placebo, and patients with contraindications to VKAs were allocated to aspirin versus placebo only. VKA was the most effective treatment (HR for VKA vs placebo for vascular death, myocardial infarction, stroke, or systemic emboli, 0.53, 0.36-0.79, with an absolute risk reduction of about $8 \%$ annually. The HR for VKA versus aspirin was $0.60(0 \cdot 41-0 \cdot 87)$, although aspirin was of some value in patients ineligible for VKAs $(0 \cdot 83,0.65-1.05)$. VKAs were more effective than aspirin at preventing recurrent stroke $(\mathrm{p}, 0.001)$. Although there was a trend towards benefit, the reduction in the annual risk of recurrent stroke with aspirin $(10 \%, n=404)$ compared with placebo (12\%, $\mathrm{n}=378)$ did not reach significance $(\mathrm{p}=0.31)$.There were more major haemorrhages with VKA, but the benefits clearly outweighed this risk [42].

The findings of EAFT were corroborated in those patients with previous TIA or ischaemic stroke who were enrolled in SPAF III [43], was designed to evaluate effectiveness of alternative therapy than high dose VKAs (with INR between 2 and 3), in high risk patients with AF. 1044 patients with $\mathrm{AF}$ and with at least one thromboembolic risk factor (congestive heart failure or left ventricular fractional shortening $<$ or $=25 \%$, previous thromboembolism, systolic blood pressure of more than $160 \mathrm{~mm} \mathrm{Hg}$ at study enrollment, or being a woman aged over 75 years) were randomly assigned either a combination of low-intensity, fixed-dose warfarin (international normalised ratio [INR] 1.2-1.5 for initial dose adjustment) and aspirin (325 mg/day) or adjusted-dose warfarin (INR 2.0-3.0). Drugs were given open-labelled. The mean INR during follow-up of patients taking combination therapy $(\mathrm{n}=521)$ was 1.3 , compared with 2.4 for those taking adjusted-dose warfarin $(\mathrm{n}=523)$. During follow-up, 54\% of INRs in patients taking combination therapy were 1.2-1.5 and $34 \%$ were less than 1.2. SPAF III trial was halted early, after a mean, follow-up of 1.1 years, because patients allocated to fixed low-dose VKA (INR 1.2-1.5) plus aspirin 325 $\mathrm{mg}$ daily had four times more ischaemic strokes during follow-up than did those allocated to regular VKA (INR 2.0 $3.0)$ at an interim analysis $(p<0.0001)$. The annual rates of disabling stroke $(5.6 \%$ vs $1.7 \%, \mathrm{p}=0.0007)$ and of primary event or vascular death $(11.8 \%$ vs $6.4 \%, \mathrm{p}=0.002)$, were also higher with combination therapy. The rates of major bleeding were similar in both treatment groups. So, Lowintensity, fixed-dose warfarin plus aspirin in this regimen is insufficient for stroke prevention in patients with nonvalvular $\mathrm{AF}$ at high-risk for thromboembolism.

The only other trial apart from EAFT run solely in patients with recent cerebral ischaemia and atrial fibrillation, comparing VKAs and indobufen, found 15\% fewer primary outcomes with VKA than with ithe antiplatelet drug [44].

The ACTIVE-W trial compared warfarin (with an INR target between 2 and 3) to combination clopidogrel $(75 \mathrm{mg}$ once daily) plus aspirin (75-100 mg daily) ramdomly assigned in 6,706 patients with AF. After a median follow-up of 1.3 years, the annual occurrence of stroke, systemic embolization, myocardial infarction, or vascular death was $3.9 \%$ in the warfarin group and $5.6 \%$ in the double antiplatelet group (RR, 1.44; 95\% CI, 1.18-1.76; $\mathrm{P}=0.0003$ ). There was no significant difference in major bleeding between the two treatment groups ( $2.4 \%$ per year for those on clopidogrel plus aspirin vs $2.2 \%$ per year on warfarin; RR 1.10 [0.831.45]) Due to a clear benefit in the warfarin group, the study was stopped prematurely, confirming how VKAs are more effective also than the association aspirin + clopidogrel [45].

A subgroup analysis of this trial addressed the hypothesis that most of the benefit of warfarin over the combination of aspirin and clopidogrel was in patients who were already taking and tolerating oral anticoagulant therapy. Results of this analysis suggest that patients who were already taking warfarin at study entry and were randomly assigned to continue oral anticoagulation therapy had a substantial reduction in vascular events compared with those on the combination of clopidogrel and aspirin. Conversely, patients who were not taking oral anticoagulants at study entry and were randomly assigned to start oral anticoagulation had a similar rate of vascular events $(4.7 \%$ vs $5.9 \%$ per year; RR 0.79 [0.53-1.18]) compared with those on the combination of clopidogrel and aspirin [45].

In this chapter, nevertheless is not tested the efficacy of VKAs, should be cited the ACTIVE-A trial, that enrolled patients with cardioembolic stroke deemed unsuitable for warfarin therapy, either due to risk of bleeding, physician judgment, or patient preference. The effects of combination aspirin and clopidogrel versus aspirin and placebo on secondary stroke prevention were compared in 7,554 patients over a mean follow-up period of 3.6 years. Only $13 \%$ of participants had history of stroke or TIA. The clopidogrel group experienced major vascular events at a rate of $6.8 \%$ per year, compared with $7.6 \%$ per year in the control group (RR, 0.89; 95\% CI, 0.81-0.98). The risk reduction was largely due to a 
significant reduction of ischemic strokes, with rates of $1.9 \%$ versus $2.8 \%$ per year in the clopidogrel and control groups, respectively (RR, $0.68 ; 95 \% \mathrm{CI}, 0.57-0.80$ ).

However, this therapeutic benefit was hampered by a high incidence of systemic and intracranial hemorrhage. Annual rates of any major bleeding in the clopidogrel and control groups, respectively, were $2.0 \%$ versus $1.3 \%$ (RR, 1.57 ; 95\% CI, 1.29-1.92) and rates of intracranial bleeding were $0.4 \%$ versus $0.2 \%$ (RR, 1.87 ; 95\% CI, 1.19-2.94) [46]. These data suggest that treating 1000 patients with AF for 1 year with clopidogrel plus aspirin prevents eight major vascular events (including two fatal and three disabling strokes) and causes seven major haemorrhages (one fatal) compared with aspirin alone.

Because of the frequent finding of co-existence in the same patients of the need of both anticoagulant and antiplatelet therapy $(\mathrm{AF}+$ ischemic heart disease, $\mathrm{AF}+$ peripheral artery disease, etc), both warfarin and aspirin are thought to be needed to prevent thrombus formation in the left atrium and arteries.

VKAs prevent the formation of fibrin-rich thrombus (socalled "red clot") associated with AF, whereas antiplatelet treatment prevents the formation of the platelet-rich thrombus (so-called "white clot") associated with arterial vascular disease. This principle applies particularly to patients with AF who present with unstable vascular disease manifest by an acute coronary syndrome, or who are undergoing vascular injury by means of percutaneous coronary or carotid inter vention or stenting, for which aspirin plus clopidogrel is recommended. For such patients with $\mathrm{AF}$, the long-term benefit-to-harm ratio of combination therapy is not known and should be left to the clinician's discretion.

However, for patients with AF who have stable vascular disease, there is no reliable evidence to indicate that adding aspirin (or clopidogrel) to warfarin is safe and effective compared with warfarin alone. Indeed, warfarin can be an effective drug for stable coronary and cerebrovascular disease, and the haemorrhage rate seems to be greater with the combination of aspirin and warfarin [47-49].

\section{CONCLUSIONS}

Data available provide the indication to long term use of VKAs as treatment of choice for secondary stroke prevention in patients who have had a recent TIA or ischaemic stroke of cardioembolic origin. If warfarin is prescribed, one should aim for a target INR between the range 2-3 to achieve the best balance between adequate secondary prevention of cardioembolic events and the risk of major haemorrhagic complications.

If warfarin is contraindicated, long-term antiplatelet treatment with aspirin is a safe, but much less effective than VKAs; alternative treatment option that should be considered pending future data on the efficacy of, and potential licensing of, direct thrombin inhibitors and other new anticoagulant compounds actually under development.

\section{CONFLICT OF INTEREST}

The authors confirm that this article content has no conflicts of interest.

\section{ACKNOWLEDGEMENTS}

Declared none.

\section{REFERENCES}

[1] Psaty BM, Manolio TA, Kuller LH, Kronmal RA, Cushman M Fried LP, White R, Furberg CD, Rautaharju PM. Incidence of and risk factors for atrial fibrillation in older adults. Circulation 1997; 96:2455-61.

[2] Rothwell PM, Algra A, Amarenco P. Medical treatment in acute and long-term secondary prevention after transient ischaemic attack and ischaemic stroke. Lancet. 2011 May 14;377(9778):1681-92.

[3] Ansell J, Hirsh J, Hylek E, Jacobson A, Crowther M, Palareti G. Pharmacology and management of the vitamin $\mathrm{k}$ antagonists: American college of chest physicians evidence-based clinical practice guidelines (8th edition). Chest. 2008;133:160S-198S.

[4] O'Reilly RA, Aggeler PM. Studies on coumarin anticoagulant drugs. Initiation of warfarin therapy without a loading dose. Circulation 1968; 38: 169-177.

[5] Van Walraven C, Hart RG, Singer DE, Laupacis A, Connolly S, Petersen P, Koudstaal PJ, Chang Y, Hellemons B. Oral anticoagulants versus aspirin in nonvalvular atrial fibrillation: An individual patient meta-analysis. JAMA 2002; 288: 2441-8.

[6] Poli D, Antonucci E, Grifoni E, Abbate R, Gensini GF, Prisco D. Bleeding risk during oral anticoagulation in atrial fibrillation patients older than 80 years. JACC 2009; 54: 999-1002.

[7] Sorensen B, Johansen P, Nielsen GL, Sorensen JC, Ingerslev J. Reversal of the international normalized ratio with recombinant activated factor VII in central nervous system bleeding during warfarin thromboprophylaxis: Clinical and biochemical aspects. Blood Coagul Fibrinolysis 2003; 14: 469-77.

[8] Hylek EM. Oral anticoagulants. Pharmacologic issues for use in the elderly. Clinics in Geriatric Medicine 2001; 17: 1-13.

[9] The Stroke Prevention in Reversible Ischemia Trial (SPIRIT) Study Group. A randomized trial of anticoagulants versus aspirin after cerebral ischemia of presumed arterial origin. Ann Neurol 1997; 42: 857-65.

[10] Mohr JP, Thompson JL, Lazar RM, et al. A comparison of warfarin and aspirin for the prevention of recurrent ischemic stroke. N Engl J Med 2001; 345: 1444-51.

[11] The ESPRIT Study Group. Medium intensity oral anticoagulants versus aspirin after cerebral ischaemia of arterial origin (ESPRIT): a randomised controlled trial. Lancet Neurol 2007; 6: 115-24.

[12] Gorter JW, for the Stroke Prevention In Reversible Ischemia Trial (SPIRIT) and European Atrial Fibrillation Trial (EAFT) study groups. Major bleeding during anticoagulation after cerebral ischemia: patterns and risk factors. Stroke Prevention In Reversible Ischemia Trial (SPIRIT). European Atrial Fibrillation Trial (EAFT) study groups. Neurology 1999; 53: 1319-27.

[13] Elkind MS. Anticoagulation for secondary stroke prevention: another nail in the coffin? Lancet Neurol 2007; 6: 97-9.

[14] Chimowitz MI, Lynn MJ, Howlett-Smith H, et al.: Comparison of Warfarin and Aspirin for Symptomatic Intracranial Arterial Stenosis. N Engl J Med 2005; 352(13): 1305-16.

[15] Bogousslavsky J, Cachin C, Regli F, et al. Cardiac sources of embolism and cerebral infarction-clinical consequences and vascular concomitants: the Lausanne Stroke Registry. Neurology 1991; 41: 855-9.

[16] Kannel WB, Benjamin EJ. Status of the epidemiology of atrial fibrillation. Med Clin North Am 2008; 92: 17-40.

[17] Brickner ME. Cardioembolic stroke. Am J Med 1996; 100: 465-74.

[18] Gage BF, Waterman AD, Shannon W, Boechler M, Rich MW, Radford MJ. Validation of clinical classification schemes for predicting stroke: results from the National Registry of Atrial Fibrillation. JAMA 2001; 285: 2864-70.

[19] European Atrial Fibrillation Trial (EAFT) Study Group. Secondary prevention in nonrheumatic atrial fibrillation after transient ischaemic attack or minor stroke. Lancet 1993; 342: 1255-62.

[20] Petersen P, Boysen G, Godtfredsen J, Andersen ED, Andersen B. Placebo-controlled, randomised trial of warfarin and aspirin for prevention of thromboembolic complications in chronic atrial fibrillation. The Copenhagen AFASAK study. Lancet 1989; 1: 175-9.

[21] The Boston area anticoagulation trial for atrial fibrillation (BAATAF) investigators. The effect of low-dose warfarin on the 
risk of stroke in patients with nonrheumatic atrial fibrillation. $\mathrm{N}$ Engl J Med 1990; 323: 1505-11.

[22] Ezekowitz MD, Bridgers SL, James KE, Carliner NH,Colling CL, Gornick CC, Krause-Steinrauf H, Kurtzke JF, Nazarian SM, Radford MJ, et al. Warfarin in the prevention of stroke associated with nonrheumatic atrial fibrillation. Veterans affairs stroke prevention in nonrheumatic atrial fibrillation investigators. $\mathrm{N}$ Engl $\mathrm{J}$ Med 1992; 327: 1406-12.

[23] Connolly SJ, Laupacis A, Gent M, Roberts RS, Cairns JA, Joyner C. Canadian Atrial Fibrillation Anticoagulation (CAFA) Study. J Am Coll Cardiol 1991 Aug; 18(2): 349-55.

[24] European Stroke Organisation (ESO) Executive Committee; ESO Writing Committee. Guidelines for management of ischaemic stroke and transient ischaemic attack 2008. Cerebrovasc Dis 2008; 25: 457-507.

[25] Furie KL, Kasner SE, Adams RJ, et al. Guidelines for the prevention of stroke in patients with stroke or transient ischemic attack: a guideline for healthcare professionals from the American Heart Association/American Stroke Association. Stroke 2011; 42: 227-76.

[26] Hart RG, Pearce LA, Aguilar MI. Meta-analysis: Antithrombotic therapy to prevent stroke in patients who have nonvalvular atrial fibrillation. Ann Intern Med 2007; 146 (12): 857-67.

[27] Saxena R, Koudstaal PJ. Anticoagulants for preventing stroke in patients with nonrheumatic atrial fibrillation and a history of stroke or transient ischaemic attack. Cochrane Database Syst Rev 2003; 3: CD000185.

[28] Singer DE, Chang U, Fang MC, et al. The net clinical benefit of warfarin anticoagulation in atrial fibrillation. Ann Intern Med 2009; 151: 297-305.

[29] Connolly SJ, Eikelboom J, Joyner C, et al. Apixaban in patients with atrial fibrillation. N Engl J Med 2011; 364: 806-17.

[30] Executive Steering Committee on behalf of the SPORTIF III Investigators. Stroke prevention with the oral direct thrombin inhibitor ximelagatran compared with warfarin in patients with non-valvular atrial fibrillation (SPORTIF III): randomized controlled trial. Lancet 2003; 362: 1691-98.

[31] SPORTIF Executive Steering Committee for the SPORTIF V Investigators. Ximelagatran vs warfarin for stroke prevention in patients with nonvalvular atrial fibrillation: a randomized trial. JAMA 2005; 293: 690-98.

[32] Connolly SJ, Ezekowitz MD, Yusuf S, et al. Dabigatran versus warfarin in patients with atrial fibrillation. N Engl J Med 2009; 361: 1139-51.

[33] Freeman JV, Zhu RP, Owens DK, et al. Cost-eff ectiveness of dabigatran compared with warfarin for stroke prevention in atrial fibrillation. Ann Intern Med 2011; 154: 1-11.

[34] ACTIVE Writing Group. Clopidogrel plus aspirin versus oral anticoagulation for atrial fi brillation in the Atrial fibrillation Clopidogrel Trial with Irbesartan for prevention of Vascular Events (ACTIVE W): a randomised controlled trial. Lancet 2006; 367: 1903-12.

[35] Diener HC, for the Executive Steering Committee of SPORTIF III and V Investigators. Stroke prevention using the oral direct throm- bin inhibitor ximelagatran in patients with non-valvular atrial fibrillation. Pooled analysis from the SPORTIF III and V studies. Cerebrovasc Dis 2006; 21: 279-93.

[36] AMADEUS Investigators. Comparison of idraparinux with vitamin $\mathrm{K}$ antagonists for prevention of thromboembolism in patients with atrial fibrillation: a randomised, open-label, non-inferiority trial. Lancet 2008; 371: 315-21.

[37] Boulander L, Kim J, Friedman M, Hauch O, Foster T, Menzin J. Patterns of antithrombotic therapy and quality of anticoagulation among patients with non-valvular atrial fibrillation in clinical practice. Int J Clin Pract 2006; 60: 258-64.

[38] Hylek EM, Evans-Molina C, Shea C, Henault LE, Regan S. Major haemorrhage and tolerability of warfarin in the fi rst year of therapy among elderly patients with atrial fi brillation. Circulation 2007; 115: 2689-96.

[39] Hart RG, Benavente O, McBride R, Pearce LA. Antithrombotic therapy to prevent stroke in patients with atrial fibrillation: a metaanalysis. Ann Intern Med 1999;131:492-501.

[40] Stroke prevention in atrial fibrillation study. Final results. Circulation $1991 ; 84$ : 527-39.

[41] Warfarin versus aspirin for prevention of thromboembolism in atrial fibrillation: Stroke prevention in Atrial Fibrillation II study. Lancet 1994; 343: 687-91.

[42] The European Atrial Fibrillation Trial (EAFT) Study Group. Optimal oral anticoagulant therapy in patients with nonrheumatic atrial fibrillation and recent cerebral ischemia. N Engl J Med 1995; 333 : $5-10$.

[43] Stroke Prevention in Atrial Fibrillation Investigators. Adjusteddose warfarin versus low-intensity, fixed-dose warfarin plus aspirin for high-risk patients with atrial fibrillation: Stroke Prevention in Atrial Fibrillation III randomised clinical trial. Lancet 1996; 348: 633-38.

[44] Morocutti C, Amabile G, Fattapposta F, et al. Indobufen versus warfarin in the secondary prevention of major vascular events in nonrheumatic atrial fibrillation. SIFA (Studio Italiano Fibrillazione Atriale) Investigators. Stroke 1997; 28: 1015-21.

[45] The ACTIVE Writing Group on behalf of the ACTIVE Investigators. Clopidogrel plus aspirin versus oral anticoagulation for atrial fibrillation in the Atrial Fibrillation Clopidogrel Trial with Irbesartan for prevention of Vascular Events (ACTIVE W): a randomised controlled trial. Lancet. 2006; 367 (9526): 1903-12.

[46] The ACTIVE Investigators. Effect of clopidogrel added to aspirin in patients with atrial fibrillation. N Engl J Med 2009; 360: 206678.

[47] Delaney JA, Opatrny L, Brophy JA, Suissa S. Drug-drug interactions between antithrombotic medications and the risk of gastrointestinal bleeding. CMAJ 2007; 177: 347-51.

[48] Gorelick PB. Combining aspirin with oral anticoagulant therapy. Is this a safe and effective practice in patients with atrial fibrillation? Stroke 2007; 38: 1652-54.

[49] Lip GYH. Don't add aspirin for associated stable vascular disease in a patient with atrial fibrillation receiving anticoagulation. BMJ 2008; 336: 614-15. 